\title{
新型荎基修饰四硫富瓦烯衍生物的合成及性质研究
}

\author{
赵邦屯*,a 刘连委 ${ }^{*}$ 李晓川 ${ }^{b}$ 渠桂荣*,

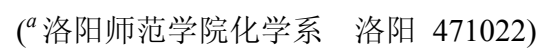 \\ ( ${ }^{b}$ 河南师范大学化学与环境科学学院 新乡 453007)
}

\begin{abstract}
摘要 在 $\mathrm{K}_{2} \mathrm{CO}_{3}$ 存在下, 对甲基苯磺酸酯四硫富瓦烯衍生物 1 和 2-荥硫酚反应合成了新型荎基修饰的四硫富瓦烯衍生 物 2, 并借助核磁、质谱、红外、元素分析等测试手段进行了结构表征. 循环伏安法研究表明化合物 2 对 $\mathrm{Zn}^{2+}$ 和 $\mathrm{Cd}^{2+}$ 有电化学响应. 荧光滴定实验表明, 加入所测的金属离子, 化合物 $\mathbf{2}$ 的荧光增强, 且 $\mathrm{Zn}^{2+}$ 和 $\mathrm{Cd}^{2+}$ 离子引起的荧光增强较 为显著. 因此, 化合物 $\mathbf{2}$ 可以作为电化学和荧光双重信号检测 $\mathrm{Zn}^{2+}$ 和 $\mathrm{Cd}^{2+}$ 的离子传感器.
\end{abstract}

关键词 四硫富瓦烯; 萎硫酚; 离子识别

\section{Synthesis and Properties of A Novel Naphthol-Substituted Tetrathiafulvalene Derivative}

\author{
Zhao, Bangtun $^{*, a} \quad$ Liu, Lianwei $^{b} \quad$ Li, Xiaochuan ${ }^{b} \quad$ Qu, Guirong* ${ }^{*, b}$ \\ ( ${ }^{a}$ Department of Chemistry, Luoyang Normal University, Luoyang 471022) \\ ( ${ }^{b}$ College of Chemistry and Environmental Science, Henan Normal University, Xinxiang 453007)
}

\begin{abstract}
A novel naphthol-substituted tetrathiafulvalene derivative 2 was conveniently synthesized and characterized by ${ }^{1} \mathrm{H}$ NMR, ${ }^{13} \mathrm{C}$ NMR, IR, MS techniques and elemental analysis. The recognition properties for $\mathbf{2}$ were carried out by CV (cyclic voltammetry) and fluorescence titration methods. The CV experiments show that compound $\mathbf{2}$ has apparent response to $\mathrm{Zn}^{2+}$ and $\mathrm{Cd}^{2+}$. Fluorescence titration experiments indicate that the fluorescence emission intensity of $\mathbf{2}$ has increased while the detected ions were added independently. Especially, while $\mathrm{Zn}^{2+}$ or $\mathrm{Cd}^{2+}$ was added, the fluorescence enhancement was more notable. Therefore, the compound 2 could serve as a dual optical-electrochemical chemosensor for $\mathrm{Zn}^{2+}$ and $\mathrm{Cd}^{2+}$ ions.
\end{abstract}

Keywords tetrathiafulvalene; naphthalene thiol; ions recognition

由于具有特有的氧化还原性质和优良的供电子能 力, 四硫富瓦烯( TTF)及其衍生物广泛应用于基于氧化 还原开关的离子传感器的研究 ${ }^{[1-3]}$. 通常对于 TTF 离子 传感器的研究主要是在 TTF 骨架上引入带有键合位点 的基团, 如冠醚、硫杂冠醚、氮杂冠醚、酰胺键、吡啶 基等, 然后通过检测离子与含有键合位点的 TTF 化合物 相互作用的电信号的变化, 从而实现对特定离子的识 别. 2009 年, 朱玉兰等 ${ }^{[4]}$ 综述了基于 TTF 阳离子化学传 感器的研究进展. 同时, 基于 TTF 阴离子化学传感器 的研究亦有报道 ${ }^{[5,6]}$. 由于识别离子的电信号变化微弱, 响应不专一, 选择性较低, 近年来, 基于 $\mathrm{TTF}$ 的光、电 传感器研究受到广泛关注, 并取得了重要进展 ${ }^{[5,7]}$. 2-萗 硫酚中包含 $\mathrm{S}$ 杂原子, 能够键合金属离子, 且具有良好
的荧光性质 ${ }^{[8 \sim 10]}$. 如果将 TTF 单元和 2-菜硫酚单元连 接起来, 有望得到具有光、电双重信号检测的离子传感 器. 本文通过简便的方法, 合成了荎基修饰的四硫富瓦 烯衍生物 2, 合成路线如 Scheme 1 所示, 并利用苂光光 谱法和电化学方法研究了其对金属离子的光、电识别行 为.

\section{1 实验部分}

\section{1 仪器与试剂}

所用试剂均为分析纯，反应和苂光实验的溶剂在使 用前均按照标准干燥方法进行处理. 化合物 $\mathbf{1}$ 参照文献 [11]合成. 仪器有 XT4A 显微熔点测定仪, 温度未校正; Bruker DPX $400 \mathrm{MHz}$ 核磁共振仪(内标: TMS); Voyager

\footnotetext{
*E-mail: zbt@lynu.edu.cn; quguir@sina.com

Received June 26 2011; revised December 25, 2011; accepted January 6, 2012.

Project supported by the National Natural Science Foundation of China (Nos. 20872058, 21172105).

国家自然科学基金(Nos. 20872058, 21172105)资助项目。
} 

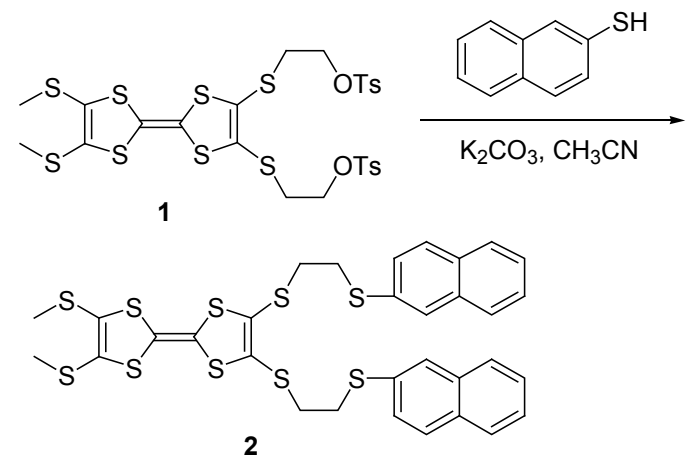

Scheme 1

MALDI-TOF 质谱仪; Nocolet 6700 傅立叶红外光谱仪; Flash 1112 元素分析仪; 上海辰华仪器公司 CHI620C 电化学工作站;日立 F-4500 荧光分光光度计.

\subsection{2,3-二[2-(2-萗基硫)-乙基硫]-6,7-二甲基硫四硫富 瓦烯(2)的合成}

氩气保护下, 将 2-菜硫酚 $(133.96 \mathrm{mg}, 0.84 \mathrm{mmol}$ )、 无水 $\mathrm{K}_{2} \mathrm{CO}_{3}(461.47 \mathrm{mg}, 3.34 \mathrm{mmol})$ 加入到含有四硫富 瓦烯 1 (253.2 mg, $0.33 \mathrm{mmol})$ 的无水乙腈 $(50 \mathrm{~mL})$ 溶液中, 回流反应 $24 \mathrm{~h}$. 停止反应后, 冷却至室温, 过滤, 并用 氯仿洗涤滤渣, 滤液旋干得粗产品, 三氯甲烷/石油醚 $(V: V=1: 5)$ 柱层析分离得黄色固体 2 , 产率为 $87.2 \%$. m.p. $93 \sim 96{ }^{\circ} \mathrm{C} .{ }^{1} \mathrm{H}$ NMR $\left(\mathrm{CDCl}_{3}, 400 \mathrm{MHz}\right) \delta$ : $7.74 \sim 7.78(\mathrm{~m}, 8 \mathrm{H}, \operatorname{ArH}), 7.40 \sim 7.46(\mathrm{~m}, 6 \mathrm{H}, \operatorname{ArH})$, $3.20 \sim 3.24\left(\mathrm{~m}, 4 \mathrm{H}, \mathrm{ArSCH}_{2}\right), 3.00 \sim 3.04\left(\mathrm{~m}, 4 \mathrm{H}, \mathrm{SCH}_{2}\right)$,

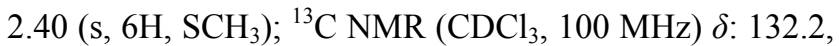
$128.9,128.2,128.1,127.8,127.8,127.7,127.4,126.8$, 126.1, 111.8, 109.7, 35.6, 33.9, 19.3; IR v: 3048, 2912, 1499, 1417, 1336, 1266, 1200, 1132, 1066, 958, 942, 891, 844, 809, 771, 761, $734 \mathrm{~cm}^{-1}$; MS (MALDI-TOF) $\mathrm{m} / z$ : $732.1\left[\mathrm{M}^{+}\right]$. Anal. calcd for $\mathrm{C}_{32} \mathrm{H}_{28} \mathrm{~S}_{10}$ : C 52.42, H 3.85; found C 52.38, H 3.88 .

\section{3 性质测定}

\subsection{1 电化学测试}

电化学实验采用标准的微型单室三电极体系, 以铂 电极 $(\phi=2 \mathrm{~mm})$ 作为工作电极, $\mathrm{Ag}-\mathrm{AgCl}$ 电极作为参比 电极, 铂丝电极作为对电极, 以 $0.1 \mathrm{~mol} \cdot \mathrm{dm}^{-3}$ 的 $n-\mathrm{Bu}_{4} \mathrm{NPF}_{4}$ 作为支持电解质, 扫描速度为 $100 \mathrm{mV} \cdot \mathrm{s}^{-1}$, 样品浓度为 $5 \times 10^{-4} \mathrm{~mol} \cdot \mathrm{dm}^{-3}$, 二氯甲烷/乙腈 $(V: V=$ $1: 1)$ 作溶剂. 每次实验前, 工作电极均用 $\mathrm{Al}_{2} \mathrm{O}_{3}$ 粉末在 鹿皮上打磨, 并用二次蒸馏水和丙酮冲洗, 干燥后使用。 测定的金属离子均为其高氯酸盐.

\subsection{2 荧光测试}

在室温条件下, 试样置于 $1 \mathrm{~cm}$ 苂光比色血中, 固定 荧光激发波长为 $269 \mathrm{~nm}$, 入射和发射狭缝为 $10 \mathrm{~nm}$, 发
射波长为 $349 \mathrm{~nm}$, 扫描荧光光谱. 样品浓度为 $2.0 \times$ $10^{-5} \mathrm{~mol} \cdot \mathrm{dm}^{-3}$, 溶剂为二氯甲烷/乙腈 $(V: V=1: 1)$, 测 定的金属离子均为其高氯酸盐.

\section{2 结果与讨论}

\section{2 化合物 2 的合成}

利用氰基的保护和去保护程序可以制备含有羟 基的 TTF 衍生物，通过磺酰化反应得到羟基活化的 TTF 衍生物 1. 在碳酸钾存在下，其和 2-菜硫酚反应得 到了富含硫原子的菜基修饰的 TTF 衍生物 $\mathbf{2}$, 方法简便, 产率较高.

\section{2 电化学性质}

由图 1 和表 1 可知，化合物 $\mathbf{2}$ 表现出 TTF 体系所 特有的两组可逆的单电子氧化还原峰(对应的阳极峰电 位和阴极峰电位之差非常接近 $0.060 \mathrm{~V}$ ). 同时, 目标化 合物 2 与 2,3,6,7-四硫甲基四硫富瓦烯模型化合物 $\mathrm{TTF}(\mathrm{SMe})_{4}$ 相比，其第一、二半波电位均发生了正移，可 能是由于 2 中 TTF 单元相连的 2-荟硫乙基的吸电子效 应，使得 TTF 中心的电子云密度降低，从而半波电位 增大.

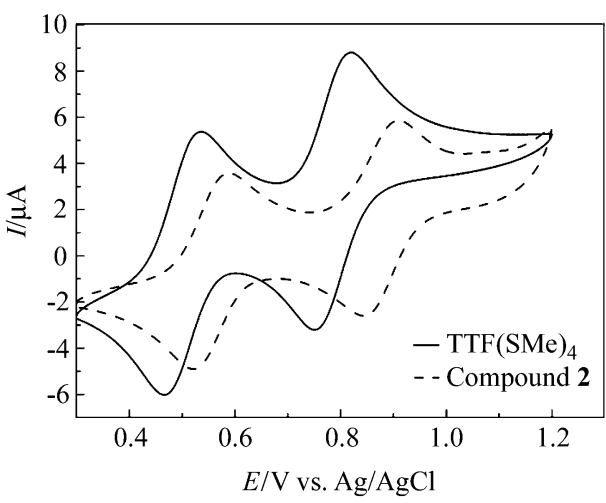

图 1 化合物 2 的循环伏安曲线

Figure 1 Cyclic voltammetry curves of compound 2

表 1 化合物 2 的氧化还原电位 $(\mathrm{V})$

Table 1 Redox potential (V) of compound 2

\begin{tabular}{ccccccc}
\hline 化合物 & $E_{1}^{\text {ox }}$ & $E_{1}^{\text {red }}$ & $E_{1 / 2}^{1}$ & $E_{2}^{\text {ox }}$ & $E_{2}^{\text {red }}$ & $E_{1 / 2}^{2}$ \\
\hline $\mathrm{TTF}(\mathrm{SMe})_{4}$ & 0.536 & 0.467 & 0.502 & 0.819 & 0.750 & 0.785 \\
$\mathbf{2}$ & 0.590 & 0.522 & 0.556 & 0.908 & 0.843 & 0.876 \\
\hline
\end{tabular}

\section{3 电化学识别性质}

从表 2 可以看出: 向化合物 2 中分别加入 3 equiv. 的 $\mathrm{Li}^{+}, \mathrm{K}^{+}, \mathrm{Pb}^{2+}, \mathrm{Mn}^{2+}$ 和 $\mathrm{Ag}^{+}$离子的高氯酸盐, 氧化还 原峰电位没有明显的变化(变化值在误差范围内); 而加 入 $\mathrm{Na}^{+}, \mathrm{Zn}^{2+}, \mathrm{Cd}^{2+}$ 离子时, 氧化还原峰电位均有明显的 负移, 这可能由于化合物 $\mathbf{2}$ 中的四个硫原子与离子发生 络合作用，降低了 2-䒬硫基的吸电子能力，使得 TTF 单 
元更容易被氧化. 并且当加入 3.0 equiv. 时, 变化达到 最大值, $\mathrm{Zn}^{2+}$ 的变化较大, $\mathrm{Na}^{+}$的变化较小. 实验表明化 合物能够选择性的识别 $\mathrm{Zn}^{2+}$ 和 $\mathrm{Cd}^{2+}$.

表 2 加入金属离子后化合物 $\mathbf{2}$ 的氧化峰电位 $(\mathrm{mV})$ 变化

Table 2 The oxidation potential changes $(\mathrm{mV})$ of compound 2 upon the added metal ions

\begin{tabular}{ccc}
\hline Added $\mathrm{M}^{n+}$ & $\Delta E_{1}^{\mathrm{ox}} / \mathrm{mV}$ & $\Delta E_{2}^{\mathrm{ox}} / \mathrm{mV}$ \\
\hline 3.0 equiv. $\mathrm{Li}^{+}$ & 1 & 1 \\
3.0 equiv. $\mathrm{Na}^{+}$ & -23 & -24 \\
3.0 equiv. $\mathrm{K}^{+}$ & 3 & 1 \\
3.0 equiv. $\mathrm{Zn}^{2+}$ & -40 & -40 \\
3.0 equiv. $\mathrm{Cd}^{2+}$ & -35 & -37 \\
3.0 equiv. $\mathrm{Pb}^{2+}$ & 3 & 3 \\
3.0 equiv. $\mathrm{Mn}^{2+}$ & -5 & -4 \\
3.0 equiv. $\mathrm{Ag}^{+}$ & -4 & -3 \\
\hline
\end{tabular}

\section{4 荧光识别性质}

加入不同金属离子, 目标化合物 $\mathbf{2}$ 在 $349 \mathrm{~nm}$ 处的苂 光强度都明显增强, 且 $\mathrm{Zn}^{2+}$ 和 $\mathrm{Cd}^{2+}$ 引起的变化较大, 见 图 2. 荧光变化的可能机理如下: 加入离子前, 化合物 $\mathbf{1}$ 中和荎环直接连接的硫原子导致分子内光诱导电子转 移(PET), 使得其荧光较弱. 当加入金属离子时, 由于配 位作用, 分子内的光诱导电子转移受阻, 荧光强度增 大, 尤其是 $\mathrm{Zn}^{2+}$ 引起的苂光增强最为显著, 而 $\mathrm{Cd}^{2+}$ 次 之. 根据 Stern-Volmer 方程 ${ }^{[12]}$, 计算知其与 $\mathrm{Zn}^{2+}$ 或 $\mathrm{Cd}^{2+}$ 的络合计量比均为 $1: 1$. 通过非线性最小二乘法 拟合 $2 \cdot \mathrm{Zn}^{2+}$ 和 $2 \cdot \mathrm{Cd}^{2+}$ 的络合常数 $\left(\lg K_{\mathrm{s}}\right)$ 分别为 3.93 和 3.54. 因此, 化合物 2 能够选择性地键合 $\mathrm{Zn}^{2+}$ 和 $\mathrm{Cd}^{2+}$. 化合物 2 与 $\mathrm{Zn}^{2+}$ 作用的荧光光谱变化和拟合见图 3.

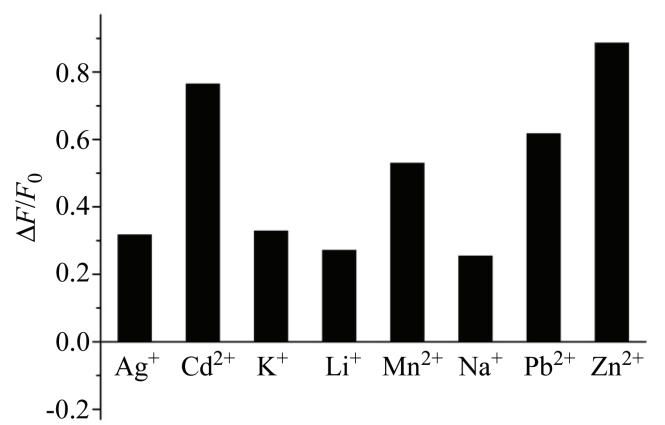

图 2 在不同离子存在的条件下化合物 $\mathbf{2}$ 在 $349 \mathrm{~nm}$ 处的荧光 强度变化 $\left(\Delta F / F_{0}\right)$

Figure 2 Fluorescence intensity changes $\left(\Delta F / F_{0}\right)$ of 2 at 349 $\mathrm{nm}$ in the presence of different metal ions

$\mathrm{CH}_{2} \mathrm{Cl}_{2} / \mathrm{CH}_{3} \mathrm{CN}(V: V=1: 1),[2]=2.0 \times 10^{-5} \mathrm{~mol} \bullet \mathrm{dm}^{-3},\left[\mathrm{M}^{n+}\right]=$ $1.0 \times 10^{-4} \mathrm{~mol} \cdot \mathrm{dm}^{-3}, \lambda_{\mathrm{ex}}=269 \mathrm{~nm}$

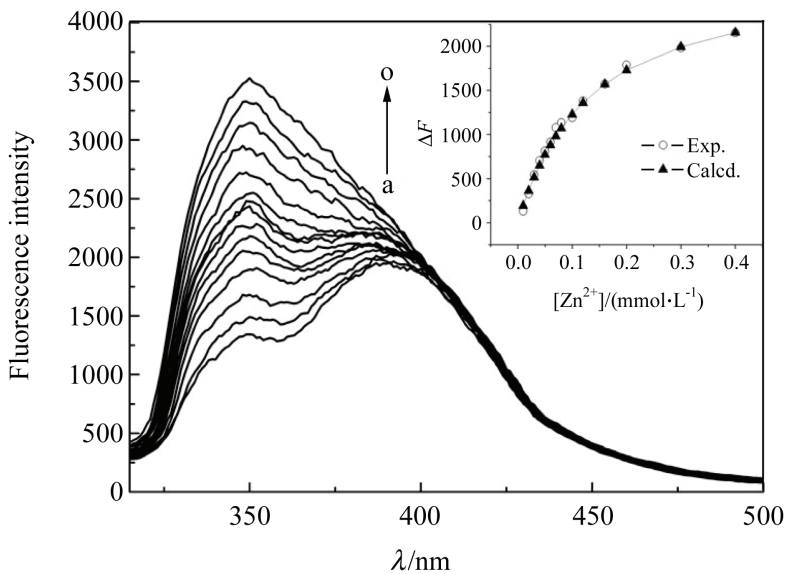

图 3 化合物 2 的 $\mathrm{CH}_{2} \mathrm{Cl}_{2} / \mathrm{CH}_{3} \mathrm{CN}(V: V=1: 1)$ 溶液中逐渐加 入 $\mathrm{Zn}\left(\mathrm{ClO}_{4}\right)_{2}\left(0 \sim 4.0 \times 10^{-4} \mathrm{~mol} \cdot \mathrm{dm}^{-3}\right.$, 从 $\mathrm{a}$ 到 $\left.\mathrm{o}\right)$ 苂光光谱的变 化

插图: 非线性最小二乘法分析苂光强度的变化来计算络合常数 $\left(\lg K_{\mathrm{s}}\right)$ $\left(\lambda_{\mathrm{ex}}=269 \mathrm{~nm}, \lambda_{\mathrm{em}}=349 \mathrm{~nm}\right)$

Figure 3 Fluorescence spectral changes of $2\left(2.0 \times 10^{-5} \mathrm{~mol} \cdot\right.$ $\left.\mathrm{dm}^{-3}\right)$ upon addition of $\mathrm{Zn}\left(\mathrm{ClO}_{4}\right)_{2}\left(0 \sim 4.0 \times 10^{-4} \mathrm{~mol} \bullet \mathrm{dm}^{-3}\right.$, from a to o) to $\mathrm{CH}_{2} \mathrm{Cl}_{2} / \mathrm{CH}_{3} \mathrm{CN}(V: V=1: 1)$

Inset: the nonlinear least-squares analysis of the differential intensity $(\Delta F)$ for determining the complex formation constant $\left(\lg K_{\mathrm{s}}\right)\left(\lambda_{\mathrm{ex}}=269 \mathrm{~nm}\right.$, $\left.\lambda_{\mathrm{em}}=349 \mathrm{~nm}\right)$

\section{3 结论}

本文设计合成了含有荎基的四硫富瓦烯衍生物 2. 电化学识别研究发现, 化合物 2 对金属离子 $\mathrm{Zn}^{2+}$ 和 $\mathrm{Cd}^{2+}$ 有响应. 苂光滴定实验表明: 加入金属离子, 2 的荧 光增强, 且 $\mathrm{Zn}^{2+}$ 和 $\mathrm{Cd}^{2+}$ 离子引起的苂光增强较为显著. 因此，目标化合物 2 可以作为电化学和荧光双重检测 $\mathrm{Zn}^{2+}$ 和 $\mathrm{Cd}^{2+}$ 的光电传感器.

\section{References}

[1] Canevet, D.; Sallé, M.; Zhang, G. X.; Zhang, D. Q.; Zhu, D. B. Chem. Commun. 2009, 17, 2245.

[2] Chen, T.; Liu, W.-J.; Cong, Z. Q.; Yin, B. Z. Chin. J. Org. Chem. 2005, 25, 570 (in Chinese).

(陈铁, 刘武军, 从志奇, 尹炳柱, 有机化学, 2005, 25, 570.)

[3] Zhao, B. T.; Zhou, Z.; Yan, Z. N.; Belhadj, E.; Derf, F. L.; Sallé, M. Tetrahedron Lett. 2010, 51, 5815.

[4] Tian, L.-B.; Zhu, Y.-L.; Yin, Q.-F.; Cao, L. Chem. Reag. 2009, 31, 895 (in Chinese).

(田礼㮏, 朱玉兰, 尹起范, 曹丽, 化学试剂, 2009, 31, 895.)

[5] Bejger, C.; Park, J. S.; Silvera, E. S.; Sessler, J. L. Chem. Commun. 2010, 46, 7745.

[6] Lee, M. H.; Cao, Q. Y.; Kim, S. K.; Sessler, J. L.; Kim, J. S. J. Org. Chem. 2011, 76, 870.

[7] Johnston, B.; Goldenberg, L. M.; Bryce, M. R.; Kataky, R. J. Chem. Soc., Perkin Trans. 2 2000, 189.

[8] Neyroz, P.; Franzoni, L.; Menna, C.; Spisni, A.; Masotti, L. J. Fluoresc. 1996, 6, 127. 
[9] Hara, S.; Tsuchie, M.; Tsujioka, R.; Kimura, M.; Fujii, M.; Kuroda, T.; Ono, N. Anal. Sci. 2000, 16, 287.

[10] Zohrehvand, S.; Evans, C. H. Polym. Int. 2005, 54, 744.

[11] John, D. E.; Batsanov, A. S.; Bryce, M. R.; Howard, J. A. K. Syn- thesis 2000, 824.

[12] Chowdhury, S.; Bhethanabotla, V. R.; Sen, R. Microsc. Microanal. 2010, 16, 725 .

(Qin, X.; Fan, Y.) 